\title{
The Epidemiological Scope of Diabetes Mellitus in District of Berat in Albania
}

\section{Alma Demo (Caka)}

\author{
Vertop Health Center Berat Albania \\ almacaka@yahoo.com
}

Doi:10.5901/ajis.2014.v3n3p483

\begin{abstract}
Early diagnosis of diabetes is one of the biggest challenges to medicine. The aim is to know the extent of diabetes epidemic through examination of records and files of patients diagnosed with diabetes and hereby present a detailed distribution of diabetes type I and type II.To valuate how the dynamics of the disease progressing clinical and laboratory aspect, what explosive factors influence, how is presented the geographical distribution of diabetes, what implications, acute or chronic, micro or macro vascular pose. Which one of these complications often meet in different types diabetes. How are diabetesspecific biological indicators as: sober glycemia 2 hours after food,HbA1c, cholesterol triglycerides, LDL, VLDL, HDL, in both types of diabetes. How are the values of biological indicators and the complications of the disease at the time of diagnosis and what happens with their values or complications during treatment with medication and dietary. To draw the attention of the employees health centers, practitioners and nurse to sensitize the population through health education on the risk of diabetes, for early diagnosis as its mostly to those patients who present with family history of diabetes, or who have the risk factor such as obesity, hypertension, smoking or alcohol. Patients are advised by health personnel for continuous measurement of glycemia, continuous monitoring of blood pressure, performing physical activities according to age and concomitant diseases, keeping a food regimen as convenient advised by a doctor to prevent obesity.
\end{abstract}

Keywords: diabetes, glycemia, HbA1c, Cholesterol, health education.

\section{Introduction}

Diabetes is a group of metabolic diseases characterized by hyperglycemia resulting from defects in insulin secretion, insulin action, or both. The chronic hyperglycemia of diabetes is associated with long-term damage, dysfunction, and failure of differentorgans, especially the eyes, kidneys, nerves, heart, and blood vessels. $\left({ }^{1}\right)$ The classifi cation of diabetes includes four clinical classes: Type 1 diabetes (results from b-cell destruction, usuallyleading toabsolute insulin deficiency) Type 2 diabetes (results from a pro- gressive insulin secretory defect on the background of insulin resistance) (2) Other specific types of diabetes due to othercauses,e.g.,geneticdefectsin b-cell function,geneticdefectsininsulinaction, diseasesoftheexocrinepancreas(suchas cysticfibrosis), and drug- or chemical-induced(suchasinthetreatmentofHIVI AIDS or after organ transplantation)( ${ }^{2}$ ) Gestational diabetes mellitus (GDM) (diabetes diagnosed during pregnancy that is not clearly overt diabetes)(2) It is possible to encounter also other types of diabetes such as genetic defects in the functionality of $\beta$ cells, genetic defects related to the inefficiency of insulin. $\left.{ }^{2}\right)\left({ }^{3}\right)$ The main factors causing diabetes are hyperlipidemia, arterial hypertension, obesity, sedentary life with no physical activity, alcohol etc. $\left.{ }^{11}\right)$ The clinic The main characteristic signs of diabetes are poliuria, polydipsia, polyphagia and weight loss. $\left(^{6}\right)\left({ }^{4}\right)$ Other clinical symptoms of diabetes are drowsiness, asthenia, and pruritus. Patients often develop different infections, and mainly they have candidose and have wounds on the skin. $\left(^{4}\right)$ People with diabetes often have urinary infections because their urine contains glucoses which has a great potential for microbial development. $\left({ }^{6}\right)\left({ }^{13}\right)$ The laboratory values of mellitus diabetes: Hyperglycemias Normal values of glycaemia are $80-120 \mathrm{mg} / \mathrm{dl}$. All other values above these values are called hyperglycemias. ${ }^{(2)} \mathrm{HbA1c}$ It is otherwise called glucated haemoglobin and its values are influenced by the chronic charge with glucoses for a period of 90-100 days. Its normal values are $4-6.5 \%\left({ }^{12}\right)$. Patients with $\mathrm{HbA1C}$ at $5.7-6.4 \%$ are considered to be at risk of developing diabetes ${ }^{12}$. If the level of $\mathrm{HbA} 1 \mathrm{c}$ is higher than $8.5 \%$ then diabetes has not been kept under control. If its value is $7 \%$ then the diabetes is balanced. If its level is less than $8.5 \%$ then diabetes is said to be controlled (12). The values of fat are represented by lipidogram where we see the cholesterol HDL,LDL,VLDL,TG.(14)(5)

The change of fat such as the increased levels of cholesterol, TG, LDL, VDL (lipoproteins with a small density) are the main reason for the acceleration of the arteriosclerotic process or macro or micro angiopathy a damage of the arteries in the big and middle calibre $\left({ }^{11}\right)$ Complications of Diabetes The more balanced diabetes is the latter the complications appear $\left(^{8}\right)$ Late complications of diabetes are: Diabetic macroangiopathy (arteriosclerosis, cardiovascular diseases) ( ${ }^{3}$ ) Diabetic microangipathy (retinopathy, nephropathy) (3) Diabetic neuropathy $\left({ }^{3}\right)$ Cure of diabetes consists in: Diet cure $\left({ }^{10}\right)$ Medical cure with oral antidiabetic $\left({ }^{10}\right)$ Cure with insulin $\left({ }^{10}\right)$ Combined cure with oral antidiabetic drugs + insulin $\left({ }^{10}\right)$ 
Physical activities depending on the age and accompanying diseases $\left({ }^{10}\right)$ Patient health education $\left({ }^{10}\right)$

The purpose This is a recognition of diabetes mellitus morbidity in the County of Berat by presenting a detailed distribution according to the geography, village, city, type of diabetes, age, gender as well as monitoring and comparing the performance of laboratory data, specific biological indicators of diabetes as sober glycaemia, 2hours after the load, $\mathrm{HbA1c}$, lipid values, triglycerides, cholesterol and lipo proteins. It also intends to introduce the presence of complications which are observed on these patients, the link which exists between lipid values, the presence of arterial hypertension, in case of receiving or not receiving treatment, implementation of nutritional diet, performance of physical activity and improving the quality of life.

The material In this research there were included 119 patients, taken in one of the suburban neighborhoods of Berat District,in a 3 -year monitoring,from January 2010 to December 2013 , aged 30-85, diagnosed with diabetes type I, type II and type II being under treatment with insulin. The study will also include other neighborhoods of the city, the town center as well, on the outskirts and in rural areas around town. This study is based on the examination of records and registers of health centers in primary health care, which have got a contract with the regional FDRSKSH (The fund of the obligatory insurances of the healthy care) which covers financially the reimbursement of the treatment of diabetes with oral anti diabetics and insulin. All patients taken in this study were enrolled in fundamental registers of chronic disease in the basic primary health care centers, where these patients have got their own records and are treated under the health insurance scheme. These patients are also enrolled in the cabinet of Endocrinology and Cardiology.

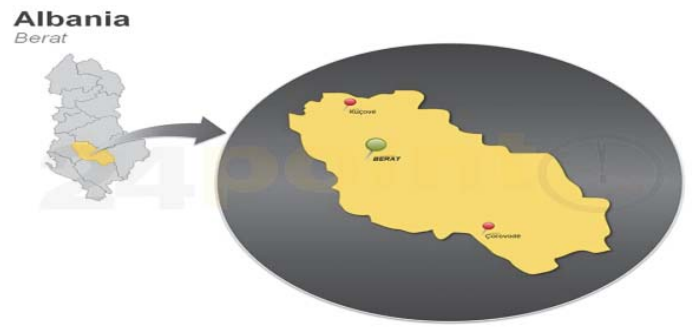

\section{Method}

There are examined all personal records of patients who were diagnosed with diabetes separated according type I, type II and type II with their medication regularly insulin. Patients appear regularly in the health center for the planned control of every month where in addition to reimbursement for their type prescription for the drugs they get such as biguanids and insulin, they also undergo the measurement of arterial hypertension, and measuring glycaemia as well. They also represent at the family doctor the results of the analyzes performed, such as lipidogram, $\mathrm{HbA1c}$, examinations conducted at the oculist doctor, ECG, which are all placed in the personal patients' recording book.

\section{Analysis of Results}

All patients taken in this study were classified according to these two type of diabetes, type I and type II. 3 patients resulted of type 1 and 116 patients resulted with type II.

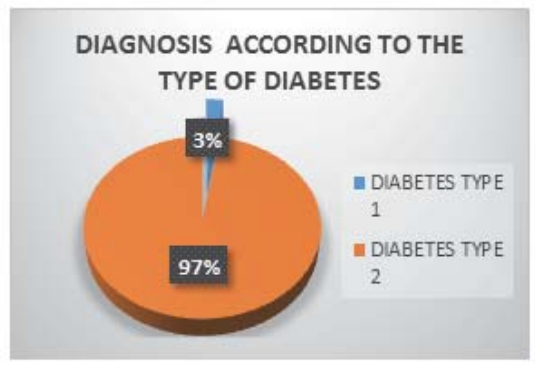

Chart 1: Classification by sex showed that 64 patients were female and 55 patients were male 


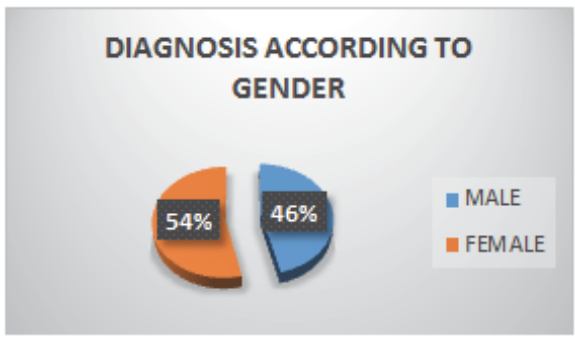

\section{Chart 2}

Depending on the treatment that they use, they are classified as follows:

Of 119 patients, 61 of them or $51.2 \%$ are treated with tablets, 55 patients or $46.2 \%$ were treated with tablets + insulin and 3 or $2.5 \%$ of them treated only with insulin.

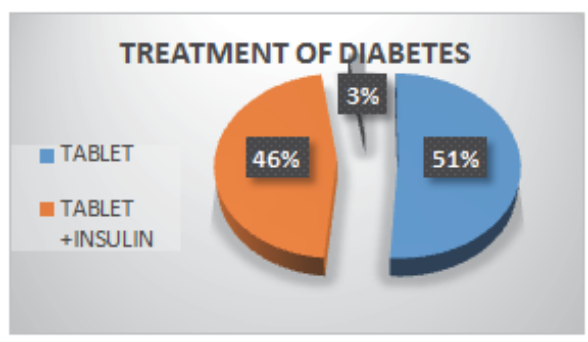

\section{Chart 3}

During the examination of the cards there were also evaluated the years these patients were suffering from diabetes. From 64 females, 17 of them or $26.5 \%$ had over 10 years who were diagnosed with diabetes and 47 of them or $73.4 \%$ had less than 10 years who were diagnosed with diabetes.

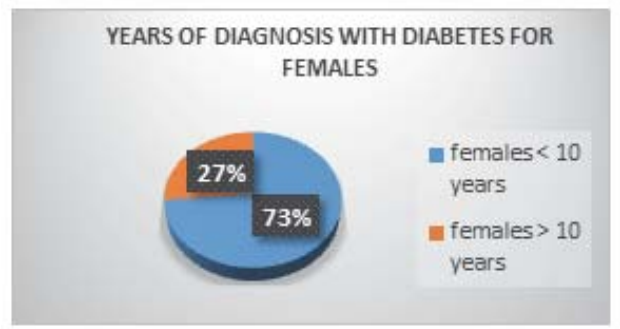

\section{Chart 4}

From 55 male patients, 16 of them or $29 \%$ were over 10 years who were diagnosed with diabetes and 39 of them or $70.9 \%$ had less than 10 years diagnosed with diabetes. 


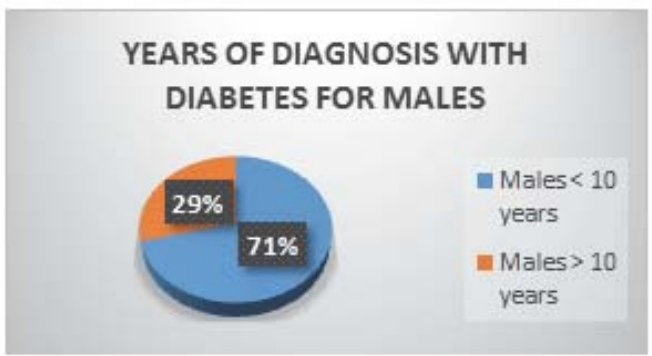

\section{Chart 5}

Average values for the period of 4 three-months of the year were compared for specific laboratory biological indicators such as sober glycaemia, 2hours after load and HbA1c for men and women both, and these results are confirmed:

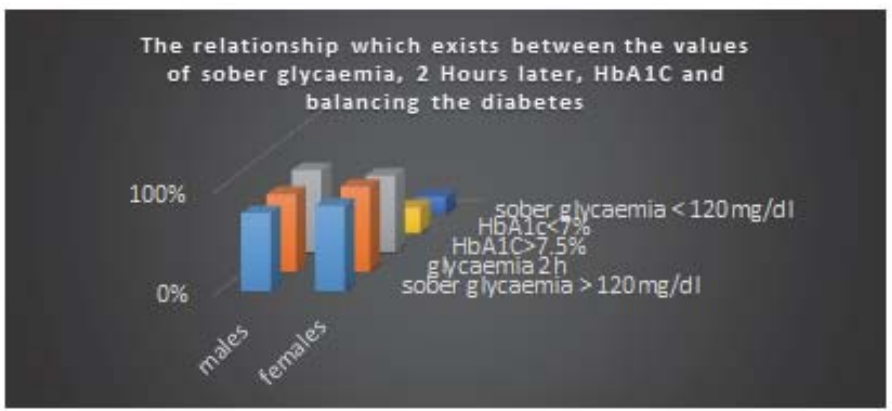

\section{Chart 6}

From 36 female patients diagnosed with diabetes of type II, 30 of them or $83.3 \%$ have values of sober glycaemia > $120 \mathrm{mg} / \mathrm{dl}$.

Of 36 women patients, 30 of them or $83.3 \%$ of them have values of glycaemia 2hours after the load $>$ than the rate.

From 36 female patients, 27 or $75 \%$ have values of $\mathrm{HbAlc}>7.5 \%$

9 female patients or $25 \%$ have values of $\mathrm{HbA} 1 \mathrm{c}<7 \%$. $120 \mathrm{mg} / \mathrm{dll}$

Of the 25 male patients diagnosed with type II of diabetes, 19 of them or $76 \%$ have the value of sober glycaemia $>$

19 of them or $76 \%$ of them have values of glycaemia $2 \mathrm{~h}$ higher than the rate.

20 of them, or $80 \%$ have got values of $\mathrm{HbA} 1 \mathrm{c}>7.5 \%$.

5 of them or $20 \%$ have values of $\mathrm{HbA} 1 \mathrm{c}<7 \%$.

From the analysis of these results it is noted that

27 female patients or $75 \%$ of them have uncontrolled diabetes

20 male patients or $80 \%$ of them have uncontrolled diabetes

Hyper glycaemia extended in time and not bringing about a balance of diabetes leads to a large number of complications. The more balanced the diabetes is, the later the complications occur and the more unbalanced the diabetes is, the sooner and the more severe the complications are presented.

During continuous monitoring of patients' personal folders we see the presence of high values of systolic and diastolic blood pressure.

There are compared the values of arterial hypertension of stage I and stage II to patients connected to their age, their gender and type of their diabetes and these results are seen: 


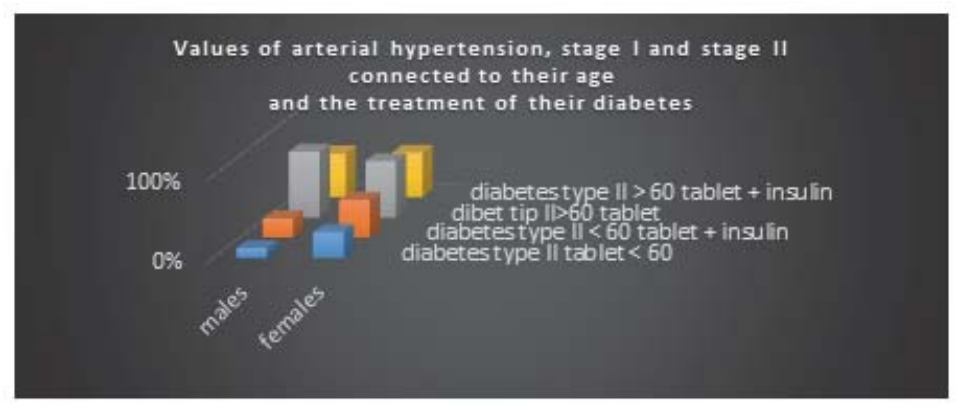

\section{Chart 7}

From 36 female patients diagnosed with type II diabetes < 60 years in medication with tablets, 32 of them or $88.8 \%$ represent values of high blood arterial pressure. From 32 female patients diagnosed with diabetes of type II, 17 of them or $31.2 \%$ have their blood pressure values of stage $\mathrm{I}$.

Of the 28 female patients diagnosed with type II of diabetes $<60$ years old in treatment with insulin + tablets, 24 of them or $85.7 \%$ represent values of high blood pressure. From 24 female patients, 11 of them or $45.8 \%$ have arterial blood pressure values of stage II.

Of the 32 female patients diagnosed with type II of diabetes $>60$ years old, in medication with tablets, 22 of them or $68.7 \%$ represent values of stage II of arterial hypertension.

From 24 female patients diagnosed with type II of diabetes $>60$ years, in treatment with insulin + tablets, 13 of them or $54.1 \%$ present values of stage II of arterial hypertension.

Of the 25 male patients diagnosed with type II of diabetes < 60 years, in medication with tablets, 3 of them or $12 \%$ of them represent values of arterial hypertension of stage I.

Of the 30 male patients diagnosed with type II of diabetes $<60$ years, in medication with insulin + tablets, 7 of them or $23.3 \%$ of them represent values of stage II of the arterial hypertension.

Of the 25 male patients diagnosed with type II of diabetes $>60$ years old, in medication with tablets, 20 of them or $80 \%$ of values of stage II of arterial hypertension.

Of the 30 male patients diagnosed with type II of diabetes $>60$ years old, in treatment with tablets + insulin, 16 of them or $53.3 \%$ represent values of stage II of arterial hypertension.

From these comparisons we see that in diabetes of type $\|<60$ year, in treatment with insulin + tablets and tablets, women dominate, that represent high values of blood pressure.

In diabetes of type II > 60 years old, men dominate with high levels of blood pressure.

We also see that with the increasing of the age of patient, we have increased values of arterial tension. HTA is a disease associated with diabetes, especially the one of type II, and it takes part in the increasing of the cardiovascular risk especially for SIZ, cerebral stroke, retinopathy and diabetic nephropathy.

Lipid values were compared with the patient's age, gender and type of diabetes, and the following results are confirmed:

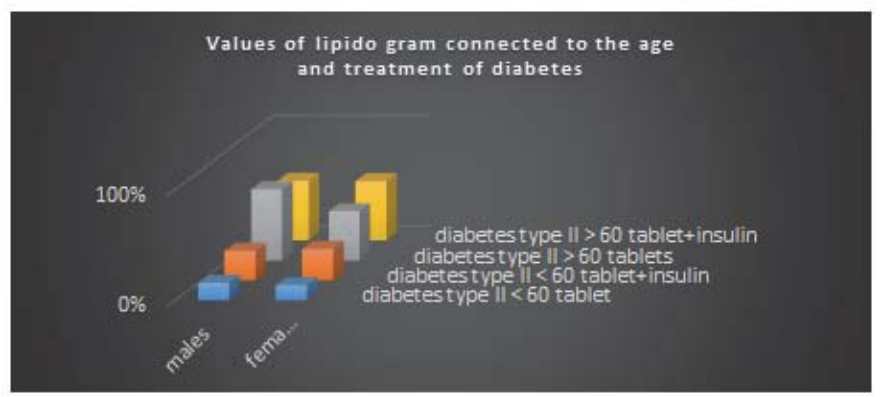

\section{Chart 8}


From 36 female patients diagnosed with type II diabetes < 60 years, in medication with tablets, 5 of them or $13.8 \%$ represent higher value of lipids.

Of the 28 female patients diagnosed with type II diabetes $<60$ years, in treatment with tablet + insulin, 8 of them or $28.5 \%$ represent higher values of lipids.

From 36 female patients diagnosed with type II diabetes > 60 years, in medication tablets, 16 of them or $44.4 \%$ represent higher values of lipids.

Of the 28 women diagnosed with type II of diabetes $>60$ years, in medication with tablets + insulin, 15 of them or $53.5 \%$ have higher lipid values .

Of the 25 male patients diagnosed with type II of diabetes $<60$ years old, in medication with tablets, 4 of them or $16 \%$ of them have high levels of lipids.

Of the 30 male patients diagnosed with type II of diabetes $<60$ years old, in medication with tablets + insulin, 8 of them or $26.6 \%$ of them have high levels of lipids.

Of the 25 male patients diagnosed with type II of diabetes $>60$ years old, in medication with tablets, 16 of them or $64 \%$ of them have high levels of lipids.

Of the 30 male patients diagnosed with type II of diabetes $>60$ years old, in medication with insulin + tablets, 16 of them or $53.3 \%$ of them have high levels of lipids.

The comparison shows that in type II of diabetes, in the medication with tablets, dominate men with higher values of lipids. It is also important the fact that with the increasing age of the patient, we have the presence of higher values of lipids. The growth of cholesterol, triglycerides and lipoproteins are the basis for accelerating the atherosclerotic process that affects arteries with large-caliber and medium one, known as atherosclerosis or diabetic macro angiopathia which is noted to patients take for survey.

It has been compared the presence of atherosclerosis or diabetic macro angiopatia conncted to the patient's age, gender and type of diabetes, and these results are confirmed:

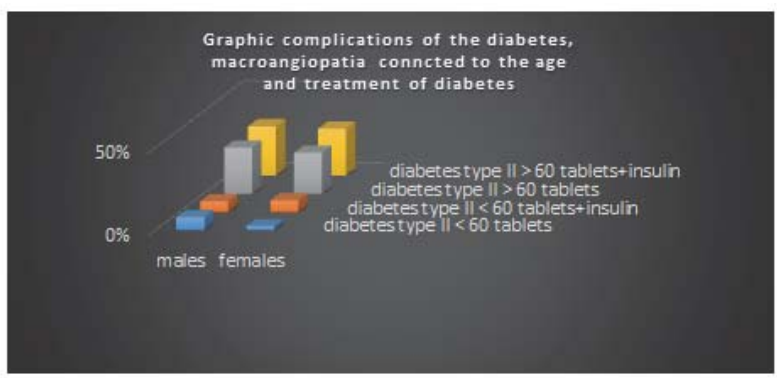

\section{Chart 9}

From 36 female patients diagnosed with type II of diabetes < 60 years old, in treatment with tablets, 1 patient or $2.7 \%$ have SKV.

Of the 28 female patients diagnosed with type II of diabetes $<60$ years old, in treatment with tablets + insulin, 2 of them insulin or $7.1 \%$ have SKV.

From 36 female patients diagnosed with type II of diabetes $>60$ years old, in treatment with tablets, 9 of them or $25 \%$ of them have macroangiopathia.

Of the 28 female patients diagnosed with type II of diabetes $>60$ years in treatment with tablets + insulin, 8 of them or $28.5 \%$ of them have macroangiopathia.

Of the 25 male patients diagnosed with type II of diabetes $<60$ years in treatment with tablets, 2 of them or $8 \%$ of them have SKV.

Of the 30 male patients diagnosed with type II of diabetes $<60$ years of age in treatment with insulin + tablets, 2 of them or $6.6 \%$ of them have SKV.

Of the 25 male patients diagnosed with type II of diabetes $>60$ years of age, in treatment with tablets, 7 of them or $28 \%$ of them have macro angiopathia.

Of the 30 male patients diagnosed with type II of diabetes $>60$ years of age, in treatment with insulin + tablets, 9 of them or $30 \%$ of them have macro angiopathia.

By comparing these results we see that in diabetes of type $\|>60$ years in age, in treatment with tablets + insulin, there are men who dominate with the presence of macro angiopathy. With the increasing of the age of patient, to the 
presence of high lipid values it is also added the risk for arteriosclerotic tiles formation in large blood vessels enabling the appearance of vascular accidents such as cerebral stroke, ischemic heart disease.

It has also been compared the presence of accompanying diseases with the type of diabetes and gender and these results are observed:

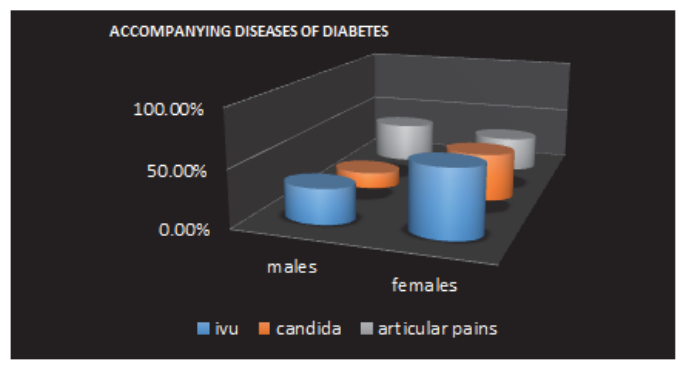

\section{Chart 10}

The first place in the accompanying diseases of diabetes to the feminine gender is occupied by IVU with $59.3 \%$, the second place is occupied by Candida with $42.1 \%$ and the third place is occupied by the articular pains with $31 \%$

To male patients, to both types of diabetes the first place in the accompanying diseases of diabetes is occupied by the articular pains with $36.3 \%$ and the second place is occupied by IVU with $30.9 \%$, and the third place is occupied by Candida with $14.5 \%$.

\section{Conclusions}

From the analysis of the above data showed that : Comparing the average values of glycaemia sober, $2 \mathrm{H}$, and type of diabetes $\mathrm{HbA1C}$ and gender showed that the 25 men diagnosed with diabetes 20 of them or $80 \%$ had uncontrolled diabetes and 36 female patients diagnosed with diabetes 27 of them or $75 \%$ had uncontrolled diabetes. In comparison that is made between blood pressure, diabetes type, age and gender showed that : In type 2 diabetes aged less than 60 years old in medication tablets and younger than 60 years old in tablets plus insulin treatment in women dominate higher present value of blood pressure. We compare lipid values with age, gender and type of diabetes that resulted in type 2 diabetes with tablets in treating older than 60 years old males predominate with higher values of lipids. Important is the fact that with increasing age patients have increased lipid values to both genders male and female.Comparing the presence makroangiopatise diabetic patient's age, gender and type of diabetes that resulted in the type 2 diabetes treatment with insulin and tablets with bigger age of 60 years old men dominate the higher presence of complications such as stroke or SZI brain by $30 \%$ The comparison of diseases associated with diabetes patient age and gender type of diabetes that resulted in first place for women diseases associated urinary tract infections. And first place occupied males articular pain . This part of the study that was presented by comparing the values of sober glycaemia, $2 \mathrm{~h}$ after the load, $\mathrm{HbA1c}$, blood pressure show a non-good situation of the appearance of diabetes, the presence of high values of cholesterol, triglycerides accompanied this by high values of glycaemia which show a negative trend in favor of developing of the complications of diabetes such as diabetic micro and macro angiopathic. The presence of hyper lipids longer time, the non-balance of the diabetes, the presence of high values of blood pressure, makes it possible the emergence of serious complications for the body such as stroke or cerebral ischemic diseases.

\section{Discussions}

According to the experts of World Health Organization, mellitus diabetes is defined as metabolic disorder caused by many reasons $\left.{ }^{1}\right)$ including the simultaneous presence of dyslipidemia, obesity and HTA with levels higher than 130-80 mmh $\left({ }^{7}\right)$. In type II diabetes, the most regular disorders are the fat disorders such as TG, cholesterol and lipoprotein disorders $\left({ }^{9}\right)$. In type II diabetes, the levels of lipids are higher and this makes it difficult the removal of triglycerides and cholesterol from the blood, enabling so the formation of arteriosclerosis plaques and as result arteriosclerosis and diabetic macroangiopathy start to develop $\left({ }^{11}\right)$. This research shows also, when comparing the specific biologic values such as glycaemia, HbA1c and lipids, that their values are higher in type II diabetes and this leads to the development of imbalanced diabetes. The more imbalanced diabetes is the faster and the graver are the complications $\left({ }^{8}\right)$. Long 
hyperglycemias keep the metabolism of fluids in general in an imbalanced condition leading to the gradual development for months and years of functional and structural changes in the tissues, organs and in the entire organism. This shows the high level of cardiovascular damages that are present in this patients as well as the presence of a series of complications at the same time. ( $\left.{ }^{3}\right)$

\section{Recommendations}

In order to enable an early diagnose of diabetes or even to keep it under control, it is of special importance to educate the population in relation to food control, the kind of food that should be eaten, number of meals, quantity and the distribution of food. The quantity of food should be controlled in respect to age and accompanying diseases. Important is to informing well the patients in relation to physical activities and the types of physical activities that are recommended by the doctor. The health personnel have a special duty in relation to:

- The identification of people with a risk of developing diabetes, mainly family members of patients with diabetes and to inform them on doing the tests regularly and controlling the arterial pressure.

- Performing regular medical checks at the family doctor or endocrinologist.

- Performing regular checks of fasting glycaemia

- Constant control of arterial pressure

- Measuring the values of lipids especially in people with a risk of developing diabetes, people with obesity or in those people with little physical activity.

- Health centres should form specialised teams with their workers in relation to educating the population 0 health.

- These health workers should develop their activities in schools, or in other places where the population usually gathers or near health centres with groups of patients at risk in order to raise their awareness for early diagnose of diabetes and the risks that it inherently carries.

\section{References}

American diabetes association(2013) Standards of medical care in diabetes-2013 diabetes care january 2013 vol. 36 no. Supplement 1S67-S74 doi: 10.2337/dc13-s067

American diabetes association(2012) Standards of medical care in diabetes-2012 diabetes care january 2012 vol. 35 no. Supplement 1 S11-S63 doi: 10.2337/dc12-s011

Masharani,U., \& German M S.(2011).Pancreatic hormones \&diabetes melitus in greenspan"s basic \& clinical endocrinology 9th edition 2011 editet by David G. Gardner Dolores Shoback chapter 17 587-597,634,636-637 issn 0891-2068

Masharani,U., \& German M S.(2011).Pancreatic hormones \&diabetes melitus in greenspan"s basic \& clinical endocrinology 9th edition 2011 editet by David G. Gardner Dolores Shoback chapter 17600 issn 0891-2068

Richard W. Nesto(2008) LDL cholesterol lowering in type 2 diabetes: What is the optimum approach? clinical diabetes january 2008 vol. 26 no. 1 8-13 doi: 10.2337/diaclin.26.1.8

American diabetes association(2013) Standards of medical care in diabetes-2013 diabetes care january 2013vol. 36 no. Supplement 1S67-S74 doi: 10.2337/dc13-s067

American diabetes association(2012) Standards of medical care in diabetes-2012 Diabetes care january 2012vol. 35 no. Supplement 1 S11-S63 doi: 10.2337/dc12-S011

Michael J. Fowler(2008) Microvascular and macrovascular complications of diabetes.Clinical diabetes april 2008 vol. 26 no. $277-82$ doi: $10.2337 /$ diaclin.26.2.77

Mary J. Malloy ,\& John P. Kane(2011) Disordes of lipoprotein metabolism in greenspan"s basic \& clinical endocrinology 9th edition 2011 editet by David G. Gardner Dolores Shoback chapter 19 675-681 issn 0891-2068

Masharani,U., \& German M S.(2011).Pancreatic hormones \&diabetes melitus in greenspan"s basic \& clinical endocrinology 9th edition 2011 editet by David G. Gardner Dolores Shoback chapter 17 593,609,612-613,617,620 issn 0891-2068

Alka M.Kanaya., \& Christian Vaisse.(2011) Obesity in greenspan"s basic \& clinical endocrinology gth edition 2011 editet by David G. Gardner Dolores Shoback chapter 20 704-705 issn 0891-2068

Masharani,U., \& German M S.(2011).Pancreatic hormones \&diabetes melitus in greenspan"s basic \& clinical endocrinology $9^{\text {th }}$ edition 2011 editet by David G. Gardner Dolores Shoback chapter 17 622-632 issn 0891-2068

Masharani,U., \& German M S.(2011).Pancreatic hormones \&diabetes melitus in greenspan"s basic \& clinical endocrinology gth edition 2011 editet by David G. Gardner Dolores Shoback chapter 17600 issn 0891-2068

Mary J. Malloy ,\& John P. Kane(2011) Disordes of lipoprotein metabolism in greenspan"s basic \& clinical endocrinology $9^{\text {th }}$ edition 2011 editet by David G. Gardner Dolores Shoback chapter 19 601,623,696,697 issn 0891-2068 